\title{
ROLE OF MULTI DETECTOR COMPUTED TOMOGRAPHY IN ASSESSMENT OF ACUTE SMALL BOWEL DISEASES
}

Khaled Mohamed Mourad Moghazy, *Galal Mohamed Moustafa Abouelnagah, Doaa Mokhtar Mohamed Emara, Ahmed Hesham Ibrahem Ame

Department of Radiodiagnosis and Intervention, *Department of Surgery, Faculty of Medicine, Alexandria University.

Introduction

One of the challenges faced by radiologists is the diagnosis of acute small bowel diseases. The small bowel is considered the narrowest, longest, and the most tortuous portion of the gastrointestinal tract which cause non-specific clinical presentations and can simulate other causes of gastrointestinal diseases. So, precise diagnosis is very important to determine the appropriate way of management. imaging modality in evaluation of acute small bowel diseases.

\section{Aim of the work}

This study was conducted to assess the role of MDCT in evaluation of acute small bowel diseases.

\section{Patients:}

This study was conducted on 38 patients; 23 males $(60.5 \%)$ and 15 emales $(39.5 \%)$ range in age from 4 years to 88 years with mean age 48 years. All patients presented clinically with acute abdominal symptoms of small bowel origin and referred to the Radio-diagnosis Department at Alexandria University Hospitals

\section{Method}

The studied patients were subjected to the following:

A-History taking and through clinical examination.

B-MDCT examination of the abdomen and pelvis including:

$$
\text { -Routine } \mathrm{CT} \text { abdomen and pelvis. }
$$$$
\text { CT entero-colonography. }
$$

C-The CT data was correlated with the surgical and medical results to reach the final diagnosis.

\section{Results}

The included patients are distributed according to the final diagnosis as the following:

A-Acute exacerbation of inflammatory bowel diseases (34.2\%).

The most frequent features are mural thickening, submucosal edema, mucosal hyper-enhancement and surrounding fat stranding. The other signs of activity include Comb sign, skip lesions and reactive mesenteric lymph nodes. Fistulae, abscess formation and inflammatory stricture are associated complications. Bilateral sacroiliitis was the associated extraintestinal manifestation.
B-Intestinal obstruction (31.6\%).

Adhesive bowel obstruction was the most common frequent cause of intestinal obstruction followed by recurrent obstructed hernia, secondary intussusception and gall stone ileus.

C-Ischemic bowel (21.1\%)

Ischemia secondary to SMV thrombus was more frequent than SMA thrombus. The CT signs included vessel filling defect (most frequent sign), mural thickening, surrounding fat stranding, mucosal hypoenhancement.

D-Perforated bowel (10.5\%).

Perforated peptic ulcer is most frequent noted cause of perforated bowel followed by traumatic perforation of small bowel. Pneumoperitoneum is the frequent $\mathrm{CT}$ finding in perforated bowel followed by small bowel mural thickening at the site of perforation with surrounding fat stranding and free fluid collection. The complication in the form of abscess formation was seen.

E-Infectious bowel diseases (2.6\%).

Distal ileal bowel loops mural thickening without mural stratification noted coupled with necrotic mesenteric lymph nodes are considered specific CT features for TB enteritis.

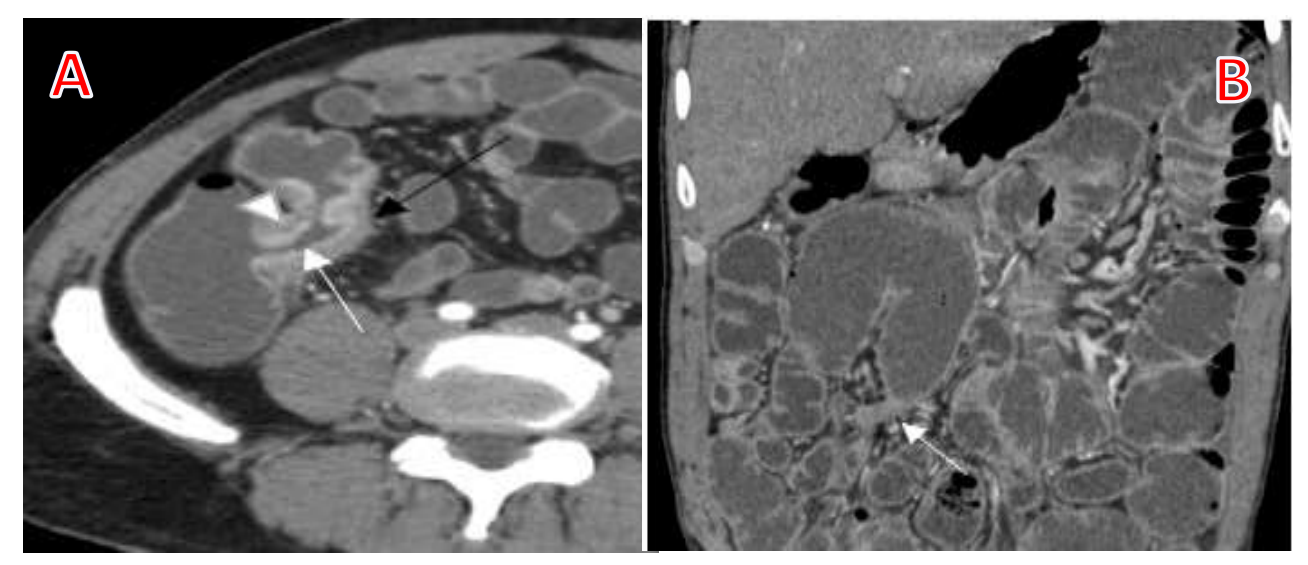

(A)Acute exacerbation of inflammatory bowel changes with mucosal enhancement, submucosal edema, and surrounding fat stranding.
(B)Adhesive small bowel

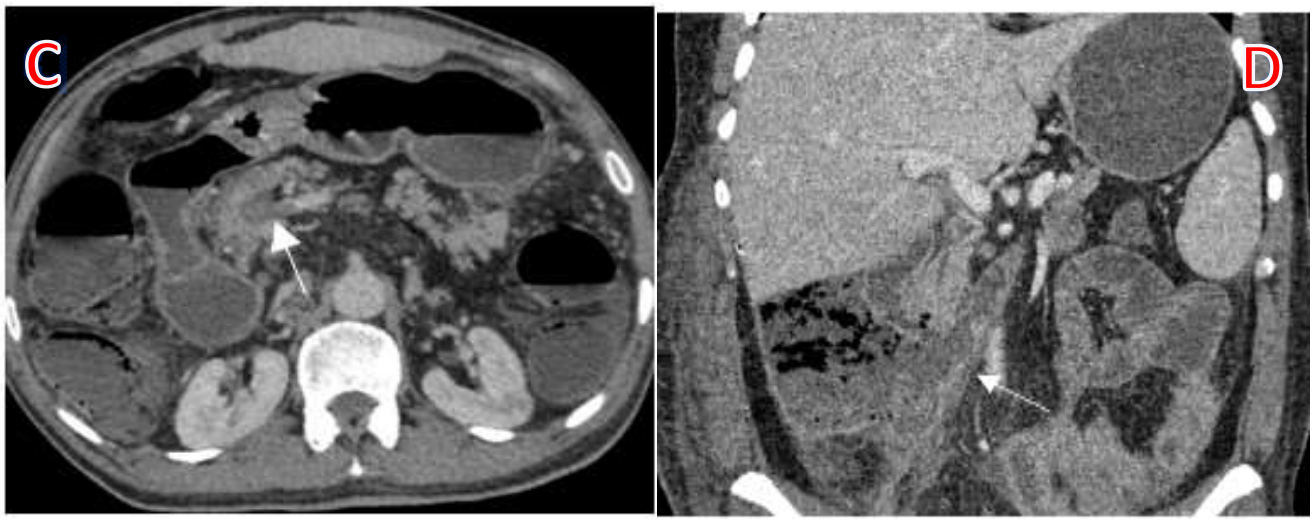

(C)Patient with SMV thrombosis.
(D) Perforated duodenum with abscess formation.

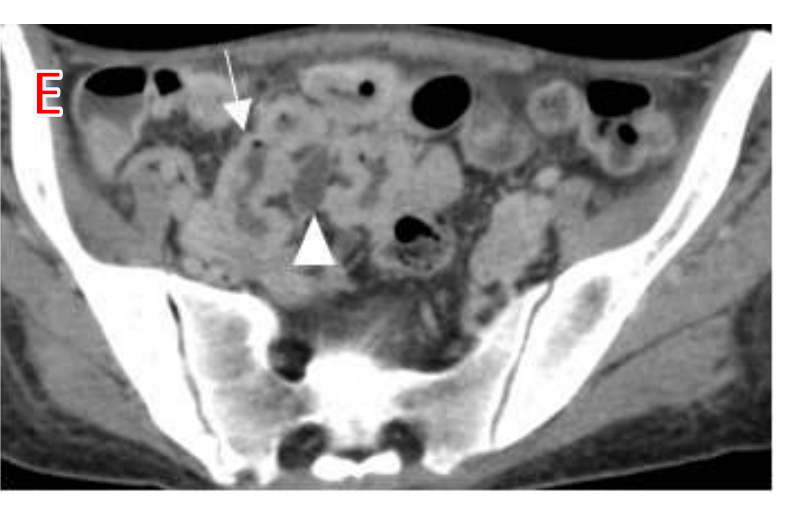

(E) TB enteritis and necrotic lymph nodes.

\section{Conclusions}

MDCT is considered a highly precise tool for the diagnosis and differentiation of acute small bowel diseases.

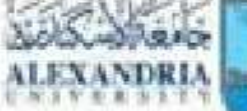

2021 (C) Alexandria Faculty of Medicine CC-BY-NC 